\title{
A highly pathogenic porcine reproductive and respiratory syndrome virus candidate vaccine based on Japanese encephalitis virus replicon system
}

\author{
Pingsheng Hu ${ }^{1}$, Xiaoming Chen ${ }^{1}$, Lihong Huang ${ }^{1}$, Shukai Liu ${ }^{1}$, Fuyu Zang ${ }^{1}$, Jinchao Xing ${ }^{1}$, Youyue \\ Zhang $^{1}{ }^{\text {, Jiaqi Liang }}{ }^{1}$, Guihong Zhang ${ }^{1}$, Ming Liao ${ }^{\text {Corresp., }}{ }^{1,2,3}$, Wenbao Qi Corresp. 1, 2,3 \\ 1 National and Regional Joint Engineering Laboratory for Medicament of Zoonoses Prevention and Control, College of Veterinary Medicine, South China \\ Agricultural University, Guangzhou, China \\ 2 Key Laboratory of Zoonoses, Key Laboratory of Animal Vaccine Development, Ministry of Agriculture, Guangzhou, China \\ 3 Key Laboratory of Zoonoses Prevention and Control of Guangdong Province, Ministry of Agriculture, Guangzhou, China
}

Corresponding Authors: Ming Liao, Wenbao Qi

Email address: mliao@scau.edu.cn, qiwenbao@scau.edu.cn

In the swine industry, porcine reproductive and respiratory syndrome (PRRS) is a highly contagious disease which causes heavy economic losses worldwide. Effective prevention and disease control is an important issue. In this study described the construction of a Japanese encephalitis virus (JEV) DNA-based replicon with a cytomegalovirus (CMV) promoter based on the genome of Japanese encephalitis live vaccine virus SA14-14-2, which is capable of offering a potentially novel way to develop and produce vaccines against a major pathogen of global health. This JEV DNA-based replicon contains a large deletion in the structural genes (C-prM-E). An PRRSV GP5/M were inserted into the deletion position of JEV DNA-based replicons to develop a chimeric replicon vaccine candidate for PRRSV. The results showed that BALB/C mice models with the replicon vaccines pJEV-REPG-2A-M-IRES and pJEV-REP-G-2A-M stimulated antibody responses and induced a cellular immune response. Analysis of ELSA data showed that vaccination with the replicon vaccine expressing GP5/M can provide better protection than traditional DNA vaccines. Therefore, the results suggested that this ectopic expression system based on JEV DNA-based replicons may represent a useful molecular platform for various biological applications, and the JEV DNA-based replicons expressing GP5/M can be further developed into a novel, safe vaccine candidate for PRRS. 
1 A highly pathogenic porcine reproductive and respiratory syndrome virus candidate vaccine based

2 on Japanese encephalitis virus replicon system

3 Pingsheng $\mathrm{Hu}^{1 *}$, Xiaoming Chen ${ }^{1 *}$, Lihong Huang ${ }^{1}$, Shukai Liu ${ }^{1}$, Fuyu Zang ${ }^{1}$, Jinchao Xing ${ }^{1}$,

4 Youyue Zhang ${ }^{1}$, Jiaqi Liang ${ }^{1}$, Guihong Zhang ${ }^{1}$, Ming Liao ${ }^{1}$, 2, 3,\# and Wenbao Qi ${ }^{1}$, 2, 3,\#

$5 \quad{ }^{1}$ National and Regional Joint Engineering Laboratory for Medicament of Zoonoses Prevention and

6 Control, College of Veterinary Medicine, South China Agricultural University, Guangzhou,

7 China.

$8 \quad{ }^{2}$ Key Laboratory of Zoonoses, Key Laboratory of Animal Vaccine Development, Ministry of

9 Agriculture, Guangzhou, China.

$10{ }^{3}$ Key Laboratory of Zoonoses Prevention and Control of Guangdong Province; Ministry of 11 Agriculture, Guangzhou, China. 
In the swine industry, porcine reproductive and respiratory syndrome (PRRS) is a highly

contagious disease which causes heavy economic losses worldwide. Effective prevention and disease control is an important issue. In this study described the construction of a Japanese encephalitis virus (JEV) DNA-based replicon with a cytomegalovirus (CMV) promoter based on the genome of Japanese encephalitis live vaccine virus SA14-14-2, which is capable of offering a potentially novel way to develop and produce vaccines against a major pathogen of global health. This JEV DNA-based replicon contains a large deletion in the structural genes (C-prM-E). An PRRSV GP5/M were inserted into the deletion position of JEV DNA-based replicons to develop a chimeric replicon vaccine candidate for PRRSV. The results showed that BALB/c mice models with the replicon vaccines pJEV-REP-G-2A-M-IRES and pJEV-REP-G-2A-M stimulated antibody responses and induced a cellular immune response. Analysis of ELSA data showed that vaccination with the replicon vaccine expressing GP5/M induced better antibody responses than traditional DNA vaccines. Therefore, the results suggested that this ectopic expression system based on JEV DNA-based replicons may represent a useful molecular platform for various biological applications, and the JEV DNA-based replicons expressing GP5/M can be further developed into a novel, safe vaccine candidate for PRRS.

\section{Introduction}

Porcine reproductive and respiratory syndrome (PRRS) is a disease that causes reproductive failures in sows and respiratory syndromes in pigs of all ages(Done \& Paton 1995). In China, the first emergence of PRRS occurred in 1995, and the causative agent PRRSV was first isolated in 1996. In 2006, a new strain of PRRSV appeared in many provinces, named highly pathogenic PRRSV (HP-PRRSV). Since then, it has been circulating and predominating in the field(Li et al. 2007; Tong et al. 2007). The GP5 protein of PRRSV (encoded by ORF5) is one of the major antigens expressed on virion surfaces and along with matrix (M) protein, are thought to be the most important targets of protective antibodies (Li \& Murtaugh 2012; Wang et al. 2007). Vaccination has been an effective way to control PRRS; and there are two main types of PRRS 
vaccines since they were first reported, modified live-attenuated vaccines (MLVs) and inactivated virus vaccines (Charerntantanakul 2012). Three commercial HP-PRRS modified live virus vaccines (HP PRRS MLVs), TJM-F92, HuN4-F112, and JXA-1R, were introduced into the Chinese swine industry, all providing good protection against HP-PRRSV infection(Leng et al. 2012; Tian et al. 2009; Yu et al. 2013). However, both kinds of the vaccine, MLVs and inactivated virus vaccines, have inherent drawbacks. Although MLVs perform well against homologous infection(Jeong et al. 2016; Linhares et al. 2012), MLVs show poor protection to heterologous strains and can-not prevent PRRSV to disseminate through the placenta (Kimman et al. 2009). Furthermore, China and Denmark each have related reports that MLVs might revert to the high virulence strain(Botner et al. 1997; Jiang et al. 2015; Opriessnig et al. 2002). Inactivated virus vaccines are safer than MLVs, but the inactivated virus vaccines provide limited protective immunity against PRRSV infection (Renukaradhya et al. 2015; Scortti et al. 2007). Moreover, more time and much more work are needed to develop both kinds of vaccine. There is an urgent need to develop vaccines against PRRS that are more effective, safe, and can be more quickly obtained, especially when new phenotypes of the PRRSV emerge.

Virus replicons hold tremendous promise as vaccine candidates because they can replicate autonomously and effectively express foreign proteins without being infectious (Kato \& Hishiki 2016).Because replicons lack the viral structural proteins genome, non-infectious replicons are as safe as a conventional inactive vaccine. At the same time, they can elicit a robust and broad reactive immune response (Aberle et al. 2005). In recent years, many flaviviruses virus replicons have been used as the expressing system for foreign genes or have been developed into vaccine candidates (Cao et al. 2011; Harvey et al. 2003). Strikingly, research has shown that the use of replicons as an expression system to create vaccines can support dual protection, both to itself and the exogenous virus (Huang et al. 2015; Yang et al. 2012).

Here, we constructed JEV DNA-based replicons with autonomous replication and effective expression of foreign proteins, which failed to generate infectious virus progeny in non- 
74 complementing cells. Moreover, we expressed GP5/M proteins of HP-PRRSV on these JEV DNA-

75 based replicons, which elicited a humoral and cellular immune response to PRRSV in a BALB/c

76 mice model. Thus, the results suggest that the JEV replicons are an efficient expression system for

77 foreign proteins, and the chimeric PRRSV vaccine based on JEV replicons could be developed as

78 a potential vaccine candidate against HP-PRRS and JEV.

79 Material and methods

80 Cell lines, virus strain, vectors, and antibody.

81 BHK-21 cells, a baby hamster kidney cell line, and 293T cells, a human embryonic kidney

82 cells line, were obtained from the Key Laboratory of Animal Disease Control and Prevention, the

83 Ministry of Agriculture, China. Cells were grown in Dulbecco's Modified Eagle Medium (DMEM;

84 Gibco, Invitrogen) supplemented with 10\% fetal bovine serum (FBS; Gibco). The JEV vaccine

85 strain SA-14-14-2 was also provided by the Key Laboratory of Animal Disease Control and

86 Prevention, the Ministry of Agriculture, China. The HP-PRRSV strain, XH-GD, is stored at the

87 Key Laboratory of Animal Disease Control and Prevention of the Ministry of Agriculture, China.

88 It was isolated in the Guangdong province in 2007 and is one of the epidemic HP-PRRSV strains

89 in Guangdong, exhibiting $99.1 \%$ nucleotide sequence identity to strain JXA1. Plasmid pEGFP-

90 N1 was purchased from Biosciences Clontech. Plasmids pIRES1-neo and pCAGGS were kindly

91 provided by ZhiGao Bu (State Key Laboratory of Veterinary Biotechnology, Harbin Veterinary

92 Research Institute, Chinese Academy of Agricultural Sciences, Harbin, China).

93 The monoclonal antibodies against the GP5 protein of PRRSV were kindly provided by

94 Researcher GuangZhi Tong (Shanghai Veterinary Research Institute, Chinese Academy of

95 Agricultural Sciences, Shanghai, China). Mouse polyclonal antibody against the NS1 protein of

96 JEV was prepared and provided by the Key Laboratory of Animal Disease Control and Prevention

97 of the Ministry of Agriculture. IRDye ${ }^{\circledR} 800$-labeled anti-mouse IgG secondary antibodies were

98 obtained from Rockland. Fluorescein isothiocyanate (FITC)-conjugated goat anti-mouse IgG were

99 obtained from SIGMA. 
100

101

102

103

104

105

106

107

108

109

110

111

112

113

114

115

116

117

118

119

120

121

122

123

124

125

\section{Plasmid constructs.}

The JEV replicons plasmid vector is depicted in Fig. 1. All the primers used to construct the recombinant plasmid are shown in Table 1 and Table 2. Fragments 5' UTR-c23,B,C, and II were amplified from the cNDA of JEV genome SA14-14-2 using primers in Table 1. The CMV promoter and fragment $\mathrm{D}$ were amplified from plasmid pEGFP-N1 using primers in Table 1. Fragment A was amplified from the CMV promoter and 5' UTR-c23 by overlap PCR using primers pCMV-F and pJEV164-olR. Then, fragments A and B were used as template to produce fragment I through overlap PCR using primers pCMV-F and pJEV5706R. Amplified fragment III using primers pJEV9117F and HDVr-pA-R in the same way as shown in Fig.1. Finally, DNA fragment I, fragment II, and fragment III were subcloned into the Low copy plasmid named pJEVREP. Fragment EGFP, both without and with the termination codon, were amplified from plasmid pEGFP-N1 using primers EGFP-F and EGFP-R or EGFP-F and EGFP-R-taa. Fragment IRES was amplified from the plasmid pIRES1-neo using primers IRES-F and IRS-588R. EGFP-IRES was amplified from the fragment IRES and fragment EGFP that includes the termination codon through overlap PCR using primers EGFP-F and IRES-588R. Finally, fragment EGFP without the termination codon and EGFP-IRES were digested by SalI and SpeI and then cloned into pJEVREP namely pJEV-REP-GFP and pJEV-REP-GFP-IRES, respectively.

Fragments GP5 (primers GP5F and GP5R-FMDV2A-R) and M (primers MF-FMDV2A-F and MR-SalI) were amplified from the PRRSV genome. Fragment G-2A-M was amplified from fragments GP5 and M through overlap PCR using primers GP5F and MR-SalI. Then, G-2A-M as a template to amplify G-2A-MR using primers GP5F and M-R. Fragments G-2A-MR and IRES were used as templates to produce G-2A-M-IRES through overlap PCR using primer GP5F and IRES-588R. Finally, G-2A-M-IRES and G-2A-M were digested by SalI and SpeI and cloned into pJEV-REP vector, named pJEV-REP-G-2A-M-IRES and pJEV-REP-G-2A-M, respectively. Using IRES-F-SpeI-TAA and IRES-588R as primers, amplified IRES-SS from pJEV-REP-G-2AM-IRES. Then, digested by SalI-HF and SpeI and cloned into a pJEV-REP vector named pJEV- 
126 REP-IRES. Fragment G-2A-M-EX was amplified from pJEV-REP-G-2A-M using primers GP5F-

127 EcoRI and MR-XhoI. Then, fragment G-2A-M-EX was digested by EcoRI and XhoI and cloned

128 into eukaryotic expression vector pCAGGS, named pCAGGS-GM. All of our restriction enzyme

129 digestion and cloning procedures were performed according to standard protocols.

130 Transfection.

131 BHK-21 and $293 \mathrm{~T}$ cells which grew to $90 \%$ confluence in 6-well cell culture plates, were

132 transfected with plasmid by Lipofectamine 2000 Reagent (Invitrogen, 11668-027) as previously

133 described (Qi et al. 2008). Briefly, $2 \mu \mathrm{g}$ of DNA was diluted in 50 $\mu$ l of serum-free Opti-MEM

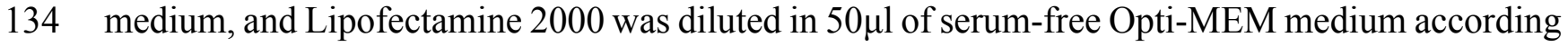

135 to the manufacturer's recommendation. We then added diluted DNA to each tube of diluted

136 Lipofectamine 2000 Reagent (1:1 ratio) and incubated it at $25{ }^{\circ} \mathrm{C}$ for $5 \mathrm{~min}$. We removed the cell

137 culture medium and washed with Opti-MEM. Then, DNA-reagent complex was added to cells. At

138 the same time, $500 \mu \mathrm{l}$ Opti-MEM also added and further cultured at $37^{\circ} \mathrm{C}$. After $6 \mathrm{~h}$, the supernatant

139 was removed, and the cells were further incubated with fresh medium with 2\% FBS for 48 h.

140 Indirect immunofluorescence assays (IFA).

141 The cells were washed with phosphate-buffered saline (PBS) and then, fixed with 4\%

142 paraformaldehyde at room temperature for $30 \mathrm{~min}$. Cell monolayers were permeabilized with $0.2 \%$

143 Triton X-100 for $10 \mathrm{~min}$. Then, incubated for $1 \mathrm{~h}$ in the presence of Mouse polyclonal antibodies

144 against the NS1 protein of JEV $(1: 100)$ in PBS buffer at $37^{\circ} \mathrm{C}$, followed by $1 \mathrm{~h}$ incubation in PBS

145 containing goat anti-mouse secondary antibodies conjugated to FITC at a dilution of 1:200. The

146 fluorescence signals were visualized using a fluorescence microscope.

147 Western Blotting.

148 Western blot analysis was performed as previously described (Qi et al. 2015). Briefly, cell 149 monolayers were incubated with RIPA lysis buffer (Beyotim, P0013B) containing 1 mM PMSF 150 (Beyotim, ST506), then centrifuged and boiled with SDS-PAGE loading buffer. Equivalent 151 Proteins sample were separated on SDS-PAGE gels and electro-transferred onto PVDF 
152 membranes (Beyotim, FFP30). After blocking, the membranes were incubated with primary

153 antibodies, PRRSV GP5 protein monoclonal antibodies, and IRDye 800-conjugated goat anti-

154 mouse as the secondary antibody. Finally, membranes were scanned using an Odyssey Imaging

155 System (Li-Cor, Amerca).

156 Immunization of mice.

157 Sixty (eight-week-old) SPF female BALB/c mice were purchased from the Guangdong

158 Medical Laboratory Animal Center (GDMLAC) and the number of production license is SCXK

159 2013-0002. BALB/c mice were assigned randomly into five groups of twelve mice each. Three

160 groups of BALB/c mice were immunized 50 $\mu$ l each hind leg with pJEV-REP-G-2A-M-IRES,

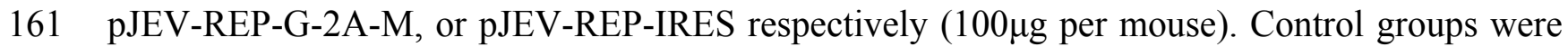

162 immunized using the same protocol with either pCAGGS-GM or PBS. Mice were immunized on

163 days 0,21 , and 42. Mice blood samples were harvested every 2 weeks, and serum samples from

164 each group were stored for virus antibody testing.

165 Enzyme Linked Immunosorbent Assay (ELISA).

166 Mouse sera were obtained from blood samples. The PRRSV antibodies level was tested using

167 the ELISA Kit (LSIVet Porcine PRRSV/US-Serum, Franc). Briefly, mouse sera were diluted 100-

168 fold, and a $100 \mu$ l sample added to the 96 plate which be pre-packaged with PRRSV components.

169 The wells were incubated for $30 \mathrm{~min}$ at $37^{\circ} \mathrm{C}$. Then, after washing with PBS, $100 \mu 1 \mathrm{HRP}$-Conjugate

170 anti-mouse antibody reagent was added to every wells, and incubated for $60 \mathrm{~min}$ at $37^{\circ} \mathrm{C}$. Washing

171 with PBS again. 50ul Chromogen Solution A and Chromogen Solution B were added and evaded

172 the light preservation for $15 \mathrm{~min}$ at $37^{\circ} \mathrm{C}$. Finally, the absorbance at $630 \mathrm{~nm}$ was read

173 after adding the stop Solution.

174 CCK-8 assay.

175 Lymphocytes were isolated from different vaccination groups of mice two weeks after third 176 immunization and treatment as previously described(Cao et al. 2011). The WST-8 dye (Beyotime 
177 Inst Biotech, China) was used to detect cell proliferation according to manufacture's instruction.

178 Briefly, lymphocytes isolated from different vaccination groups mice. The lymphocytes were 179 adjusted to a working concentration of $1 \times 10^{6}$ cells $/ \mathrm{ml}$ in RPMI-1640 and $100 \mu$ lymphocytes were 180 added into 96-well plate respectively. Then, $100 \mu l$ concentration of $200 \mathrm{TCID}_{50} / \mathrm{ml}, \mathrm{XH}-\mathrm{GD}$,

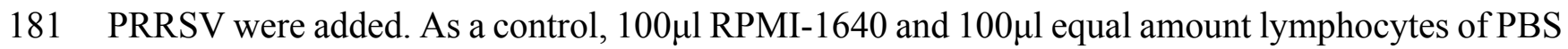
182 control group were added into another $96-$ well plate. Incubated the plates at $37^{\circ} \mathrm{C}$ in a $5 \% \mathrm{CO}_{2}$ 183 incubator. Then, $10 \mu \mathrm{l}$ WST-8 dye was added to each well and the 96-well plates were incubated 184 at $37^{\circ} \mathrm{C}$ for $3 \mathrm{~h}$. The asorbance was determined at $450 \mathrm{~nm}$ using a microplate reader (Thermo 185 Scientific). Calculated the stimulation index (SI) on behalf of Proliferation of the T lymphocyte, 186 which is the ratio of $\mathrm{OD}_{450} \mathrm{~nm}$ of stimulated wells to $\mathrm{OD}_{450} \mathrm{~nm}$ of unstimulated ones.

\section{7 plus or minus detection replicon RNA.}

188 The cells were harvested at the indicated times post transfection and total RNA was extracted 189 using TaKaRa MiniBEST Universal RNA Extraction Kit (TaKaRa). The primer 2403F was used

190 as an antisense primer and the primer $2609 \mathrm{R}$ as a sense reverse transcription, then $85^{\circ} \mathrm{C}$ 191 deactivation. The primers 2403F and 2609R (shown in Table 2) target a region within the E gene. 192 After reverse transcription, the primer 2403F was used to selectively quantitate the sense strand 193 and used primer 2609R to selectively quantitate the antisense strand by PCR.

194 Statistical analysis.

195 For all the data, the mean value, the standard error of the mean (SEM), and statistical analyses 196 were performed using both the unpaired t test and paired t test to determine the statistical 197 significance $(\mathrm{P}<0.05)$ between the two indicated test groups (GraphPad Prism 5.01 software).

\section{Ethics statement and Biosafety.}

199 Sixty (eight-week-old) SPF female BALB/c mice were purchased from the Guangdong 200 Medical Laboratory Animal Center (GDMLAC) and the number of production license is SCXK 201 2013-0002. All experiments were carried out in ABSL-3 facilities in compliance with the biosafety 202 committee of South China Agriculture University (SCAU) protocols. All animal experiments were 
203 reviewed and approved by the Institutional Animal Care and Use Committee at SCAU and were

204 carried out in accordance with the approved guidelines. All animal experiments were reviewed and

205 approved by the Institutional Animal Care and Use Committee at SCAU and were carried out in

206 accordance with the approved guidelines.

207 RESULTS

\section{Construction and characterization of JEV replicons.}

209 We constructed a DNA-based replicons vector, pJEV-REP, from the JEV SA-14-14-2 strain.

210 pJEV-REP contains the JEV genome sequence with a large in frame deletion (nucleotides 165-

211 2402) in the viral genome, corresponding to the middle portion of structure proteins, C-prM-E.

212 The overall strategy for constructing the JEV replicon is outlined in Fig. 1A. The sequences of

213 ribozyme HDVr and polyA had been added at the end of 3'UTR to insure that transcript RNA

214 correctly cleaved within complete 3'UTR sequence. For convenience in inserting the necessary

215 foreign genes, the SpeI and SalI restriction sites were introduced into the deletion position of the

216 structure protein. Because those restriction enzymes have many isocaudomers(Sun et al. 2013).

217 To investigate the function of JEV replicons, two different versions of the replicons vectors

218 were constructed, as shown in Fig. 1B and Fig. 1C. The replicons vector, pJEV-REP-GFP, was

219 modified by inserting reporter gene, EGFP, into the SpeI and SalI restriction sites (Fig. 1B), while

220 pJEV-REP-GFP-IRES was constructed by inserting the EGFP- IRES gene and introducing a

221 termination codon and an initiation codon at the end of EGFP and the front of

222 E25,respectively(Fig. 1C).

\section{The efficiency and replication of JEV replicons.}

224 To investigate the replication of JEV replicons, pJEV-REP-GFP-IRES and pJEV-REP-GFP 225 were transfected into $293 \mathrm{~T}$ cells, and $48 \mathrm{~h}$ post transfection we applied indirect immune 226 fluorescence analysis (IFA) to monitor viral protein NS1 synthesis. The results of IFA showed that 227 IFA staining was readily detected (Fig. 2A). To facilitate replication monitoring and to explore the 228 possibility of expressing foreign genes in the JEV replicons, the EGFP was used as a report gene 
229

230

231

232

233

234

235

236

237

238

239

240

241

242

243

244

245

246

247

248

249

250

251

252

253 254

and plasmids pJEV-REP-GFP-IRES and pJEV-REP-GFP was transfected into $293 \mathrm{~T}$ cells and BHK-21cells, respectively. Following 48h post transfection, more EGFP-expressing cells were detected in pJEV-REP-GFP-IRES than in pJEV-REP-GFP and the fluorescence intensity increased as time went by (Fig. 2B).In addition, the RT-PCR was performed to detect the replication of replicon RNA. The RT-PCR results showed increased levels of both plus- and minus-sense RNA, while no specific bands can be detected in the group without RT step (Fig. 3). Furthermore, when we diluted the cDNA at 10 times and 20 times, the specific bands amplified from plus-sense RNA were significantly brighter than those of minus-sense RNA. These results indicate that JEV replicons plasmids pJEV-REP-GFP-IRES and pJEV-REP-GFP replicated efficiently in transfected cells and an excess amount of plus-sense RNA was synthesized relative to the minus-sense RNA.

\section{Generation of recombinant JEV replicons expressing GP5/M protein.}

The JEV replicon constructed above was used for the generation of recombinant vectors expressing the GP5/M gene of PRRSV. The nucleotide sequence of the HP-PRRSV strain, XHGD, ORF5 encoding GP5 protein (almost 22KD) and ORF6 encoding M protein (almost 19KD) were amplified from the HP-PRRSV strain XH-GD genome. A DNA fragment encoding 2A protease of the foot-and-mouth disease virus (FMDV-2A) was inserted between proteins GP5 and M. Fragments G-2A-M and G-2A-M-IRES were cloned into the pJEV-REP vector and named pJEV-REP-G-2A-M and pJEV-REP-G-2A-M-IRES, respectively (Fig. 4A). The G-2A-M gene was also cloned into a eukaryotic expression vector, pCAGGS, named pCAGGS-GM to be used as control. To verify the GP5/M protein expression, pJEV-REP-G-2A-M-IRES or pJEV-REP-G2A-M was transfected into 293T cells and transfected cells were harvested and analyzed by IFA and western blot. IFA results showed that NS1 was expressed (Fig. 4B), and Western-blot analysis showed that in the pJEV-REP-G-2A-M, pJEV-REP-G-2A-M-IRES and pCAGGS-GM, the fusion protein were expressed in $293 \mathrm{~T}$ cells successfully and in negative control the fusion protein were not detect (Fig. 4C). 
255

256

257

258

259

260

261

262

263

264

265

266

267

268

269

270

271

272

273

274

275

276

277

278

279

280

Cellular immune response induced by recombinant JEV replicons expressing GP5/M protein.

To characterize the cell-mediated immune response induced by the JEV replicons vaccine, mice were killed 8 weeks after the primary immunization, and the splenocyte proliferation-based WST-8 assay was performed. WTS-8 assay indicated that the lymphocyte proliferation of mice immunized with pJEV-REP-G-2A-M-IRES or pJEV-REP-G-2A-M was higher than those immunized with pCAGGS-GM (DNA vaccine), but not significantly (Fig.5). Lymphocyte proliferation of the three immunized groups, pJEV-REP-G-2A-M-IRES, pJEV-REP-G-2A-M and pCAGGS-GM, were significantly high than the control groups pJEV-REP-IRES or PBS. These results indicate that immunization with the JEV DNA-based replicon vaccine induces lymphocyte proliferation in mice.

\section{PRRSV-specific antibodies elicited by recombinant JEV replicons expressing GP5/M protein} in mice.

Sera were harvested from mice at $0,2,4,6,8$, and 10 weeks after the first immunization and PRSSV-specific antibodies were analyzed using ELISA. As shown in (Fig. 6), mice immunized with pJEV-REP-G-2A-M-IRES or pJEV-REP-G-2A-M could produce higher PRRSV specific antibodies than those immunized with pCAGGS-GM 2 weeks after the first immunization, and the specific antibodies of the three groups immunized with pJEV-REP-G-2A-M-IRES or pJEV-REPG-2A-M or pCAGGS-GM peaked 2 weeks after the third immunization. Anti-PRRSV specific antibodies titers were significantly $(\mathrm{P}<0.05)$ higher in immune groups pJEV-REP-G-2A-M-IRES and pJEV-REP-G-2A-M than in the control groups pJEV-REP-IRES or PBS 6 weeks after first vaccination, but the pCAGGS-GM group was non-significant $(\mathrm{P}>0.05)$. However, Anti-PRRSV specific antibodies titers were significantly $(\mathrm{P}<0.05)$ higher in all three immune groups, pJEVREP-G-2A-M-IRES, pJEV-REP-G-2A-M, and pCAGGS-GM, than in the control groups pJEVREP-IRES or PBS at 8 weeks after the first immunization. Although the antibody level declined, anti-PRRSV specific antibodies titers were also significantly $(\mathrm{P}<0.05)$ higher in the immune 
281 groups pJEV-REP-G-2A-M-IRES and pJEV-REP-G-2A-M than in the control groups pJEV-REP-

282 IRES or PBS at 10 weeks after the first immunization.

283 Discussion

284 In the swine industry today, HP-PRRS is considered to be one of the most challenging 285 diseases. Vaccination has been an effective method of controlling PRRS ever since it was reported 286 (Pileri \& Mateu 2016). However, both MLVs and inactivated virus vaccines have inherent 287 drawbacks. Furthermore, like other (+) RNA viruses, PRRSV is easy to mutate and recombine. In 288 2013-2014, a new HP-PRRSV strain emerged in China with a very different genetic background 289 than the classic Chinese HP-PRRSV strains. It is a NADC30-like PRRSV strain recently 290 introduced from North America that has undergone genetic exchange with the classic HP-PRRSV 291 strains (Zhao et al. 2015), and the occurrence of attenuated strains reverting to high virulence strains 292 has been reported(Jiang et al. 2015). Thus, it is necessary to develop effective, safe, and quickly 293 obtained vaccines to protect against PRRSV, especially when new PRRS strains emerge.

294 The GP5 and M proteins are two kinds of structure proteins of the PRRSV, and they were 295 associated in hetero dimeric complexes on the surface of PRRSV (Mardassi et al. 1996). The GP5 296 protein and M protein have been shown to induce antibodies and high production of IFN$297 \beta$ (Binjawadagi et al. 2016). Here, we describe the construction of JEV replicons and use JEV 298 replicon vectors expressing the PRRSV GP5 and M proteins as a bivalent seedlings vaccine. We 299 generate two kinds of PRRS vaccine, named pJEV-REP-G-2A-M-IRES and pJEV-REP-G-2A-M.

300 In order to release the GP5 and M proteins from the JEV replicon polyprotein, the foot-and-mouse

301 disease virus 2A autoprotease sequence was inserted between the GP5 and M genes. IFA with

302 JEV-NS1 polyclonal antibody show that the JEV replicon vector can replicate effectively (Fig.

303 4B). Western-blot analysis showed that GP5/M proteins were also expressed successfully in 304 vitro.(Fig. 4C). Splenocyte proliferation was an important point in detecting a cell-mediated 305 immune response. The result has demonstrated the ability of JEV replicon vaccine to induce 306 splenocyte proliferation following the final immunization. The ability to induce PRRSV specific 
307 immune responses was detected by ELISA. The research showed that the intramuscular 308 immunization of mice with the JEV vaccine induced special anti-PRRSV antibodies after the first 309 inoculation. With subsequent immunization boosting, the level of specific anti-PRRSV antibody 310 increased and finally peaked 2 weeks after the third immunization. The result showed that the 311 antibody levels of immune groups pJEV-REP-G-2A-M-IRES and pJEV-REP-G-2A-M were 312 higher than immune group pCAGGS-GM all the time, and the antibodies of immune group 313 pCAGGS-GM decreased faster than the JEV replicon vaccine group. Furthermore, in the pJEV314 REP-IRES vaccinated group, the level of special anti-PRRSV antibodies were the same as in the 315 PBS group. Meanwhile, the ELISA method is very accuracy and the immune response is 316 specifically directed against PRRS proteins. We can infer that the PRRS proteins GP5 and M were 317 responsible for the observed immune response rather than the backbone of the JEV replicon. Of 318 course, the ELISA method is very accuracy. However, an additional control with pJEV-REP-IRES 319 plasmid expressing an irrelevant protein can be worth to take into account to rule out the possibility 320 that any protein different from GP5 or M induces antibodies that cross-react with PRRSV antigens. 321 All the data showed that the JEV replicon vaccine induced effective antibody against PRRSV. 322 However, the protection capability of the JEV replicon vaccines against PRRS needs more assay 323 in pigs though, previous report showed that Pigs immunized with CSF-JE VRP replicon vaccine 324 displayed strong antibody responses and protection against CSFV and JEV challenge 325 infections(Yang et al. 2012). In the aspect of preventing JEV infection, it is report that the JEV 326 replicon vaccine could confer protection to itself (Huang et al. 2015). Therefore, we focus on the 327 immune protection to PRRS rather than JEV. Of course, this genetic engineering vaccine can be 328 further optimized to enhance the immune protective effect for example, to screen appropriate 329 immune adjuvant for JEV replicon vaccine or inoculation of animals with suitable methods. At the 330 same time, this vaccine with JEV replicons expressing GP5/M proteins induced a systemic 331 immune responses to PRRS, it is can be a good heterologous Prime-Boost HP-PRRS Vaccination

332 Regimens. The JEV replicon vaccine can be used for the first immunization and the commercial 
333 HP-PRRS vaccines can be used to improve anti-PRRSV immunity. Because it is a laborious work

334 to attenuate PRRSV and it takes a long time to develop a new modified live-attenuated vaccine

335 when a new variation of PRRS emerges, this genetically engineered vaccine is good emergency

336 supplement strategy.

337 Flaviviruses replicons, characterized by their high efficiency in expressing heterologous 338 genes without producing infectious progeny virus, are useful tools for understanding the 339 replication of viruses and exploring antiviral screening, and it is also applied as a potential 340 expression system to be an antivirus vaccine candidate(Cao et al. 2011; Suzuki et al. 2014).

341 Replicons vaccines has several advantages over inactivated vaccines and many subunit vaccines.

342 It can prepared more quickly than inactivated virus vaccines, and replicons vaccines can enhance

343 cross-protection through the fusion of expressing antigenic peptides of different strains(Sun et al.

344 2016). Compared with the baculovirus expression system, replicon vaccines are more convenient

345 to operate since they do not need to express antigens by cell culture and be purified. Otherwise,

346 the baculovirus expression system is liable to fail in vivo because of the complement system of

347 host(Tani et al. 2003). Therefore, we constructed JEV DNA-based replicons through deleting the

$348 \mathrm{C}$, prM, E encoding region which replicates autonomously but fails to generate infectious virus

349 progeny in non-complementing cells; therefore, it can be used as an expression system for foreign 350 proteins. In this study, the nucleotides from 165-2402, corresponding to most of the structural 351 proteins $\mathrm{C}$, prM, E, were deleted in JEV replicons. The $\mathrm{N}$-terminal 23 amino acids of the $\mathrm{C}$ protein,

352 have been reported as performing the essential role of the cis-acting element and in regulating 353 minus sense RNA synthesis(also known as containing the cyclization sequence)(Khromykh et al.

354 2001). Compared with other JEV replicons, we retained less of the nucleic acid sequence of C

355 protein, and the IFA and RT-PCR results showed that our constructed JEV replicon can self356 replicate effectively (Fig. 2). We also retained the C-terminal 25 amino acids of E protein to 357 preserve the correct processing and translocation of NS1 and the remaining nonstructural 358 polyprotein in the correct topology across the membrane of the endoplasmic reticulum( $\mathrm{Ng}$ et al. 
359 2007). At the same time, we constructed JEV DNA-based replicons with a cytomegalovirus

360 (CMV) promoter. Compared with RNA-based replicons, DNA-based replicons operate more

361 conveniently and are more stable in the form of plasmids. Furthermore, host cells are more likely

362 to intake DNA-based replicons (Cao et al. 2011; Varnavski et al. 2000).

\section{Conclusions}

364 In conclusion, we describe the construction of JEV replicon with deletion in the C, prM, E

365 encoding region, which can be used as an efficient expression system for foreign proteins. In

366 addition, this JEV replicon used to express GP5/M proteins which showed better immunogenicity

367 compared with tradition DNA vectors expressing GP5/M proteins. These results indicate that our

368 JEV replicons are a useful molecular platform for expressing foreign proteins capable of inducing

369 a protective immune response and could serve as a promising strategy to develop as a potential

370 bivalent seedlings vaccine candidate.

371 Acknowledgments

372 We would like to thank the National and Regional Joint Engineering Laboratory for

373 Medicament of Zoonoses Prevention and Control providing HP-PRRSV strain. Also, we would

374 like to hank Harbin Veterinary Research Institute providing plasmids. Moreover, we would like to

375 thank Shanghai Veterinary Research Institute providing antibodies.

376 All authors reviewed and revised the first and final drafts of this manuscript.

\section{Reference}

378

379

380

381

382

383

384

385

Aberle JH, Aberle SW, Kofler RM, and Mandl CW. 2005. Humoral and cellular immune response to RNA immunization with flavivirus replicons derived from tick-borne encephalitis virus. J Virol 79:15107-15113. 10.1128/JVI.79.24.15107-15113.2005

Binjawadagi B, Lakshmanappa YS, Longchao Z, Dhakal S, Hiremath J, Ouyang K, Shyu DL, Arcos J, Pengcheng S, Gilbertie A, Zuckermann F, Torrelles JB, Jackwood D, Fang Y, and Renukaradhya GJ. 2016. Development of a porcine reproductive and respiratory syndrome virus-like-particle-based vaccine and evaluation of its immunogenicity in pigs. Arch Virol 161:1579-1589. 10.1007/s00705-016-2812-0

Botner A, Strandbygaard B, Sorensen KJ, Have P, Madsen KG, Madsen ES, and Alexandersen S. 1997. Appearance of 
386

387

388

389

390

391

392

393

394

395

396

397

398

399

400

401

402

403

404

405

406

407

408

409

410

411

412

413

414

415

416

417

418

419

420

421

422

423

424

acute PRRS-like symptoms in sow herds after vaccination with a modified live PRRS vaccine. Vet Rec 141:497-499.

Cao F, Li XF, Yu XD, Deng YQ, Jiang T, Zhu QY, Qin ED, and Qin CF. 2011. A DNA-based West Nile virus replicon elicits humoral and cellular immune responses in mice. $J$ Virol Methods 178:87-93. 10.1016/j.jviromet.2011.08.018

Charerntantanakul W. 2012. Porcine reproductive and respiratory syndrome virus vaccines: Immunogenicity, efficacy and safety aspects. World J Virol 1:23-30. 10.5501/wjv.v1.i1.23

Done SH, and Paton DJ. 1995. Porcine reproductive and respiratory syndrome: clinical disease, pathology and immunosuppression. Vet Rec 136:32-35.

Harvey TJ, Anraku I, Linedale R, Harrich D, Mackenzie J, Suhrbier A, and Khromykh AA. 2003. Kunjin virus replicon vectors for human immunodeficiency virus vaccine development. J Virol 77:7796-7803.

Huang YT, Liao JT, Yen LC, Chang YK, Lin YL, and Liao CL. 2015. Japanese encephalitis virus replicon-based vaccine expressing enterovirus-71 epitope confers dual protection from lethal challenges. J Biomed Sci 22:74. 10.1186/s12929-015-0181-8

Jeong J, Choi K, Kang I, Park C, and Chae C. 2016. Evaluation of a 20year old porcine reproductive and respiratory syndrome (PRRS) modified live vaccine (Ingelvac((R)) PRRS MLV) against two recent type 2 PRRS virus isolates in South Korea. Vet Microbiol 192:102-109. 10.1016/j.vetmic.2016.07.006

Jiang YF, Xia TQ, Zhou YJ, Yu LX, Yang S, Huang QF, Li LW, Gao F, Qu ZH, Tong W, and Tong GZ. 2015. Characterization of three porcine reproductive and respiratory syndrome virus isolates from a single swine farm bearing strong homology to a vaccine strain. Vet Microbiol 179:242-249. 10.1016/j.vetmic.2015.06.015

Kato F, and Hishiki T. 2016. Dengue Virus Reporter Replicon is a Valuable Tool for Antiviral Drug Discovery and Analysis of Virus Replication Mechanisms. Viruses 8. 10.3390/v8050122

Khromykh AA, Meka H, Guyatt KJ, and Westaway EG. 2001. Essential role of cyclization sequences in flavivirus RNA replication. J Virol 75:6719-6728. 10.1128/JVI.75.14.6719-6728.2001

Kimman TG, Cornelissen LA, Moormann RJ, Rebel JM, and Stockhofe-Zurwieden N. 2009. Challenges for porcine reproductive and respiratory syndrome virus (PRRSV) vaccinology. Vaccine 27:3704-3718. 10.1016/j.vaccine.2009.04.022

Leng X, Li Z, Xia M, He Y, and Wu H. 2012. Evaluation of the efficacy of an attenuated live vaccine against highly pathogenic porcine reproductive and respiratory syndrome virus in young pigs. Clin Vaccine Immunol 19:1199-1206. 10.1128/CVI.05646-11

Li J, and Murtaugh MP. 2012. Dissociation of porcine reproductive and respiratory syndrome virus neutralization from antibodies specific to major envelope protein surface epitopes. Virology 433:367-376. 10.1016/j.virol.2012.08.026

Li Y, Wang X, Bo K, Wang X, Tang B, Yang B, Jiang W, and Jiang P. 2007. Emergence of a highly pathogenic porcine reproductive and respiratory syndrome virus in the Mid-Eastern region of China. Vet $J$ 174:577-584. 10.1016/j.tvjl.2007.07.032

Linhares DC, Cano JP, Wetzell T, Nerem J, Torremorell M, and Dee SA. 2012. Effect of modified-live porcine reproductive and respiratory syndrome virus (PRRSv) vaccine on the shedding of wild-type virus from an infected population of growing pigs. Vaccine 30:407-413. 10.1016/j.vaccine.2011.10.075 
425 Mardassi H, Massie B, and Dea S. 1996. Intracellular synthesis, processing, and transport of proteins encoded by 426 ORFs 5 to 7 of porcine reproductive and respiratory syndrome virus. Virology 221:98-112. 10.1006/viro.1996.0356

Ng CY, Gu F, Phong WY, Chen YL, Lim SP, Davidson A, and Vasudevan SG. 2007. Construction and characterization of a stable subgenomic dengue virus type 2 replicon system for antiviral compound and siRNA testing. Antiviral Res 76:222-231. 10.1016/j.antiviral.2007.06.007

431

Opriessnig T, Halbur PG, Yoon KJ, Pogranichniy RM, Harmon KM, Evans R, Key KF, Pallares FJ, Thomas P, and Meng XJ. 2002. Comparison of molecular and biological characteristics of a modified live porcine reproductive and respiratory syndrome virus (PRRSV) vaccine (ingelvac PRRS MLV), the parent strain of the vaccine (ATCC VR2332), ATCC VR2385, and two recent field isolates of PRRSV. J Virol 76:11837-11844.

Pileri E, and Mateu E. 2016. Review on the transmission porcine reproductive and respiratory syndrome virus between pigs and farms and impact on vaccination. Vet Res 47. Artn 108

Qi WB, Hua RH, Yan LP, Tong GZ, Zhang GH, Ren T, Wu DL, and Liao M. 2008. Effective inhibition of Japanese encephalitis virus replication by small interfering RNAs targeting the NS5 gene. Virus Res 132:145-151. 10.1016/j.virusres.2007.11.014

Qi WB, Tian J, Su S, Huang LH, Li HN, and Liao M. 2015. Identification of potential virulence determinants associated H9N2 avian influenza virus PB2 E627K mutation by comparative proteomics. Proteomics 15:1512-1524. 10.1002/pmic.201400309

Renukaradhya GJ, Meng XJ, Calvert JG, Roof M, and Lager KM. 2015. Inactivated and subunit vaccines against porcine reproductive and respiratory syndrome: Current status and future direction. Vaccine 33:3065-3072. 10.1016/j.vaccine.2015.04.102

Scortti M, Prieto C, Alvarez E, Simarro I, and Castro JM. 2007. Failure of an inactivated vaccine against porcine reproductive and respiratory syndrome to protect gilts against a heterologous challenge with PRRSV. Vet $\operatorname{Rec} 161: 809-813$.

Sun Q, Liu J, Li Y, Zhang Q, Shan S, Li X, and Qi B. 2013. Creation and validation of a widely applicable multiple gene

Suzuki R, Ishikawa T, Konishi E, Matsuda M, Watashi K, Aizaki H, Takasaki T, and Wakita T. 2014. Production of single-

Sun D, Khatun A, Kim WI, Cooper V, Cho YI, Wang C, Choi EJ, and Yoon KJ. 2016. Attempts to enhance cross-protection against porcine reproductive and respiratory syndrome viruses using chimeric viruses containing structural genes from two antigenically distinct strains. Vaccine. 10.1016/j.vaccine.2016.06.069 round infectious chimeric flaviviruses with DNA-based Japanese encephalitis virus replicon. J Gen Virol 95:60-65. 10.1099/vir.0.058008-0

Tani H, Limn CK, Yap CC, Onishi M, Nozaki M, Nishimune Y, Okahashi N, Kitagawa Y, Watanabe R, Mochizuki R, Moriishi K, and Matsuura Y. 2003. In vitro and in vivo gene delivery by recombinant baculoviruses. $J$ Virol 77:9799-9808. 10.1128/Jvi.77.18.9799-9808.2003

462

Tian ZJ, An TQ, Zhou YJ, Peng JM, Hu SP, Wei TC, Jiang YF, Xiao Y, and Tong GZ. 2009. An attenuated live vaccine 463 based on highly pathogenic porcine reproductive and respiratory syndrome virus (HP-PRRSV) protects 
464 piglets against HP-PRRS. Vet Microbiol 138:34-40. 10.1016/j.vetmic.2009.03.003

465 Tong GZ, Zhou YJ, Hao XF, Tian ZJ, An TQ, and Qiu HJ. 2007. Highly pathogenic porcine reproductive and respiratory $466 \quad$ syndrome, China. Emerg Infect Dis 13:1434-1436. 10.3201/eid1309.070399

467 Varnavski AN, Young PR, and Khromykh AA. 2000. Stable high-level expression of heterologous genes in vitro and in 468 vivo by noncytopathic DNA-based Kunjin virus replicon vectors. J Virol 74:4394-4403.

469 Wang SP, Fang LR, Fan HY, Jiang YB, Pan YF, Luo R, Zhao Q, Chen HC, and Xiao SB. 2007. Construction and 470 immunogenicity of pseudotype baculovirus expressing GP5 and M protein of porcine reproductive and respiratory syndrome virus. Vaccine 25:8220-8227. 10.1016/j.vaccine.2007.09.069

471

472

Yang Z, Wu R, Li RW, Li L, Xiong Z, Zhao H, Guo D, and Pan Z. 2012. Chimeric classical swine fever (CSF)-Japanese

473 encephalitis (JE) viral replicon as a non-transmissible vaccine candidate against CSF and JE infections. Virus

474 Res 165:61-70. 10.1016/j.virusres.2012.01.007

475

Yu X, Chen N, Deng X, Cao Z, Han W, Hu D, Wu J, Zhang S, Wang B, Gu X, and Tian K. 2013. Genomic sequencing 476 reveals mutations potentially related to the overattenuation of a highly pathogenic porcine reproductive and respiratory syndrome virus. Clin Vaccine Immunol 20:613-619. 10.1128/CVI.00672-12

478

Zhao K, Ye C, Chang XB, Jiang CG, Wang SJ, Cai XH, Tong GZ, Tian ZJ, Shi M, and An TQ. 2015. Importation and 479 Recombination Are Responsible for the Latest Emergence of Highly Pathogenic Porcine Reproductive and Respiratory Syndrome Virus in China. J Virol 89:10712-10716. 10.1128/Jvi.01446-15 
482 FIG. 1. Schematic drawing of replicons. (A) Strategy to construct the JEV replicon. An in-frame 483 deletion in the structural region of C-prM-E was from nt 165 to 2402 and SpeI and SalI site was 484 generated at the junction of the deletion position. (B and C) Schematic drawing of replicons contain 485 report gene. (B) The EGFP fragment without a termination code and initiation code was engineered 486 into the deletion position at SpeI and SalI. (C) The IRES-EGFP fragment, containing a termination 487 code at the end of EGFP and an initiation code at the front of E25, which was engineered into the 488 deletion position at the SpeI and SalI site.

489 FIG. 2. The efficiency and replication of JEV replicons. (A) 293T cells transfected with replicon 490 plasmids pJEV-REP-GFP-IRES or pJEV-REP-GFP were subjected to IFA at the indicated time 491 points post transfection. JEV-NS1 polyclonal antibody and FITC-conjugated goat anti-mouse IgG 492 antibody were used as primary and secondary antibodies for IFA, respectively. (B) BHK-21 cells 493 and 293T cells were transfected with pJEV-REP-GFP-IRES or pJEV-REP-GFP and monitored the 494 EGFP signals by fluorescence microscope at $48 \mathrm{~h}$ post transfection.

495 FIG. 3. Plus or minus detection of replicon RNA. (A) BHK-21 cells were transfected with replicon 496 plasmids pJEV-REP-GFP-IRES or pJEV-REP-GFP. Total RNA isolated at $48 \mathrm{~h}$ post transfection 497 was subjected to RT-PCR analysis with JEV-specific primers. RT reaction was performed under 498 standard conditions with avian myeloblastosis virus (AMV) reverse transcriptase (Takara), Primer $4992403 \mathrm{~F}$ and $2609 \mathrm{R}$ targeting a region within the E gene, was used as a sense or an antisense primer 500 respectively in RT step to selectively quantitate either minus- or plus-sense replicon RNA. (B) The 501 cDNA samples obtained from B were diluted with 10 times or 20 times and quantitate either minus502 or plus-sense replicon RNA by PCR.

503 FIG. 4. Expression of PRRSV GP5/M protein in JEV replicons. (A) Schematic drawing of pJEV504 REP-G-2A-M and pJEV-REP-G-2A-M-IRES. (B) 293T cells transfected with replicon plasmids 505 pJEV-REP-G-2A-M and pJEV-REP-G-2A-M-IRES were subjected to IFA at the indicated time 506 points post transfection. JEV-NS1 polyclonal antibody and FITC-conjugated goat anti-mouse IgG 
508 analyze the GP5 and M protein expression, PRRSV GP5 protein monoclonal antibodies were used

509 as primary antibodies and IRDye 800-conjugated goat anti-mouse as the secondary antibody. 293T

510 cells transfected with pCAGGS-GM was used as a positive control while 293T cells were used as

511 a negative control. Equivalent proteins sample were added in per lane. The GP5 protein almost

$51222 \mathrm{KD}$ and the M protein almost 19KD. The fusion protein almost 41KD was showed by red arrow.

513 FIG. 5. Lymphocyte proliferation elicited by recombinant JEV replicons expressing GP5/M

514 protein in mice. Eight-week-old, SPF BALB/c mice were immunized with pJEV-REP-G-2A-M-

515 IRES, pJEV-REP-G-2A-M, pCAGGS-GM, pJEV-REP-IRES, and PBS respectively.

516 Lymphocytes were separated from the spleens of mice two weeks after the third immunization.

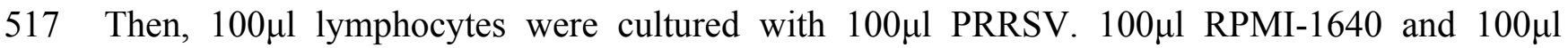

518 lymphocytes of PBS control group were added into another 96-well plate as a control. After 84h,

519 CCK-8 assay was performed to detect the lymphocyte proliferation. Stimulation Index $(\mathrm{SI})=$

$520 \mathrm{OD}_{450} \mathrm{~nm}(\mathrm{PRRSV}) / \mathrm{OD}_{450} \mathrm{~nm}$ (Control). Statistical were compared the pCAGGS-GM, pJEV-

521 REP-G-2A-M-IRES and pJEV-REP-G-2A-M groups with the pJEV-REP-IRES group, 522 respectively.

523 FIG. 6. Specific antibodies elicited by recombinant JEV replicons expressing GP5/M protein in 524 mice. Eight-week-old, SPF BALB/c mice were immunized with pJEV-REP-G-2A-M-IRES, 525 pJEV-REP-G-2A-M, pCAGGS-GM, pJEV-REP-IRES, and PBS respectively. Mice sera were 526 collected at indicated time points after immunization and used to detect the GP5 specific antibodies 527 by ELISA. 
Table $\mathbf{1}$ (on next page)

Primers used for construction of the subgenomic replicons of JEV. 
1 Table. 1 Primers used for construction of the subgenomic replicons of JEV.

\begin{tabular}{|c|c|}
\hline primer & Sequences $\left(5^{\prime}-3^{\prime}\right)$ \\
\hline pCMV-F & TTTTTGGCGGCCGCTAGTTATTAATAGTAATCAATTACGG \\
\hline \multirow[t]{2}{*}{ pCMV-R } & GTTCACACAGATAAACTTCTGGATCTGACGGTTCACTAAA \\
\hline & CCAGCTCTG \\
\hline \multicolumn{2}{|l|}{ 5'UTR-c23 } \\
\hline pJEV1-F & AGAAGTTTATCTGTGTGAACTTCTTGG \\
\hline \multirow[t]{2}{*}{ pJEV164-olR } & CTCGGTCGACGGTGGTAACACTAGTGCGGGGTAGGCCGC \\
\hline & GTTTCAGC \\
\hline \multicolumn{2}{|l|}{ Fragment B } \\
\hline \multirow[t]{2}{*}{ PJEV2403-olF } & $\underline{\text { ACTAGTGTTACCACCGTCGACCGAGACCGATCAATTGCTT }}$ \\
\hline & TGG \\
\hline pJEV5706R & TTACGCTCGCCACAAACCAC \\
\hline \multicolumn{2}{|l|}{ Fragment II } \\
\hline pJEV-5557F & CGACCCCGCCTGGAACCACG \\
\hline pJEV-9155R & GAACCCCAAAGCTTCAAACTCTAGA \\
\hline \multicolumn{2}{|l|}{ Fragment $\mathrm{C}$} \\
\hline pJEV9117F & CTTGGAGCACGGTATCTAGAGTTTG \\
\hline \multirow[t]{2}{*}{ pJEV10976R } & AGCCGGCGCCAGCGAGGAGGCTGGGACCATGCCGGCCAT \\
\hline & CAGGAGATCCTGTGTTCTTCCTCACCAC \\
\hline \multicolumn{2}{|l|}{ Fragment D } \\
\hline \multirow[t]{2}{*}{ HDVr-pA-F } & CCTGATGGCCGGCATGGTCCCAGCCTCCTCGCTGGCGCCG \\
\hline & GCTAACTTGTTTATTGCAGCTTA \\
\hline HDVr-pA-R & CGA $\underline{\text { GGTACCTTGTCCAAACTCATCAATGTATCTTA }}$ \\
\hline
\end{tabular}

2 Lined part represent for restriction enzymes. 


\section{Table 2 (on next page)}

Primers used for construction of different plasmid and primers used for plus or minus detection of replicon RNA. 
1 Table. 2 Primers used for construction of the subgenomic replicons of JEV.

\begin{tabular}{|c|c|}
\hline primer & Sequences $\left(5^{\prime}-3^{\prime}\right)$ \\
\hline EGFP-F & CCGCACTAGTATGGTGAGCAAGGGCGAGG \\
\hline EGFP-R & CTCGGTCGACCTTGTACAGCTCGTCCATGC \\
\hline \multirow[t]{2}{*}{ EGFP-R-taa } & GGGGGAGGGAGAGGGGCGTTACTTGTACAGCTCGTCC \\
\hline & ATGCC \\
\hline IRES-F & CGCCCCTCTCССТСCСCC \\
\hline IRES-588R & 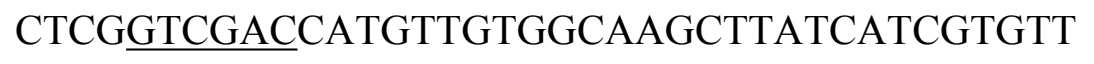 \\
\hline $2403 \mathrm{~F}$ & CGAGACCGATCAATTGCTTTGG \\
\hline 2609R & CTTCGCTAGGGATCTGGGCGTTTCTGG \\
\hline GP5F & 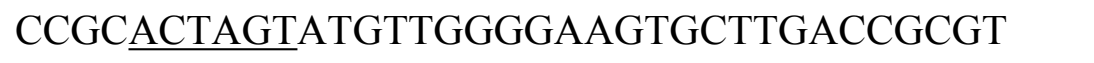 \\
\hline \multirow[t]{2}{*}{ GP5R-FMDV2A-R } & CTCAACGTCTCCCGCCAACTTGAGGAGGTCGAAGTTCA \\
\hline & GAAGCTGGAGACGACCCCATTGTTCTGCT \\
\hline \multirow[t]{2}{*}{ MF-FMDV2A-F } & GACCTCCTCAAGTTGGCGGGAGACGTTGAGTCCAACCC \\
\hline & TGGGCCTATGGGGTCGTCTCTAGACGA \\
\hline MR-SalI & 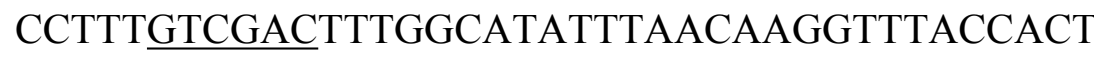 \\
\hline \multirow[t]{2}{*}{ M-R } & GGGGGAGGGAGAGGGGCGTTATTTGGCATATTTAACA \\
\hline & AGGTTTACCACT \\
\hline GP5F-EcoRI & CCTTTEAATTCATGTTGGGGAAGTGCTTGACCGCGT \\
\hline \multirow[t]{2}{*}{ MR-XhoI } & CСTTTCTCGAGTTATTTGGCATATTTAACAAGGTTTACC \\
\hline & $\mathrm{ACT}$ \\
\hline IRES-F-SpeI-TAA & CCGCACTAGTTAACGCCCCTCTCCCTCCCCC \\
\hline
\end{tabular}

2 Lined part represent for restriction enzymes. 


\section{Figure 1}

Schematic drawing of replicons.

(A) The numbers are the nucleotide positions based on the sequence of JEV SA-14-14-2 strain. An in-frame deletion in the structural region of C-prM-E was from nt 165 to 2402 and Spel and Sall site was generated at the junction of the deletion position.

(B) The EGFP fragment without a termination code and initiation code was engineered into the deletion position at Spel and Sall.

(C) The IRES-EGFP fragment, containing a termination code at the end of EGFP and an initiation code at the front of E25, which was engineered into the deletion position at the Spel and Sall site . ?

A
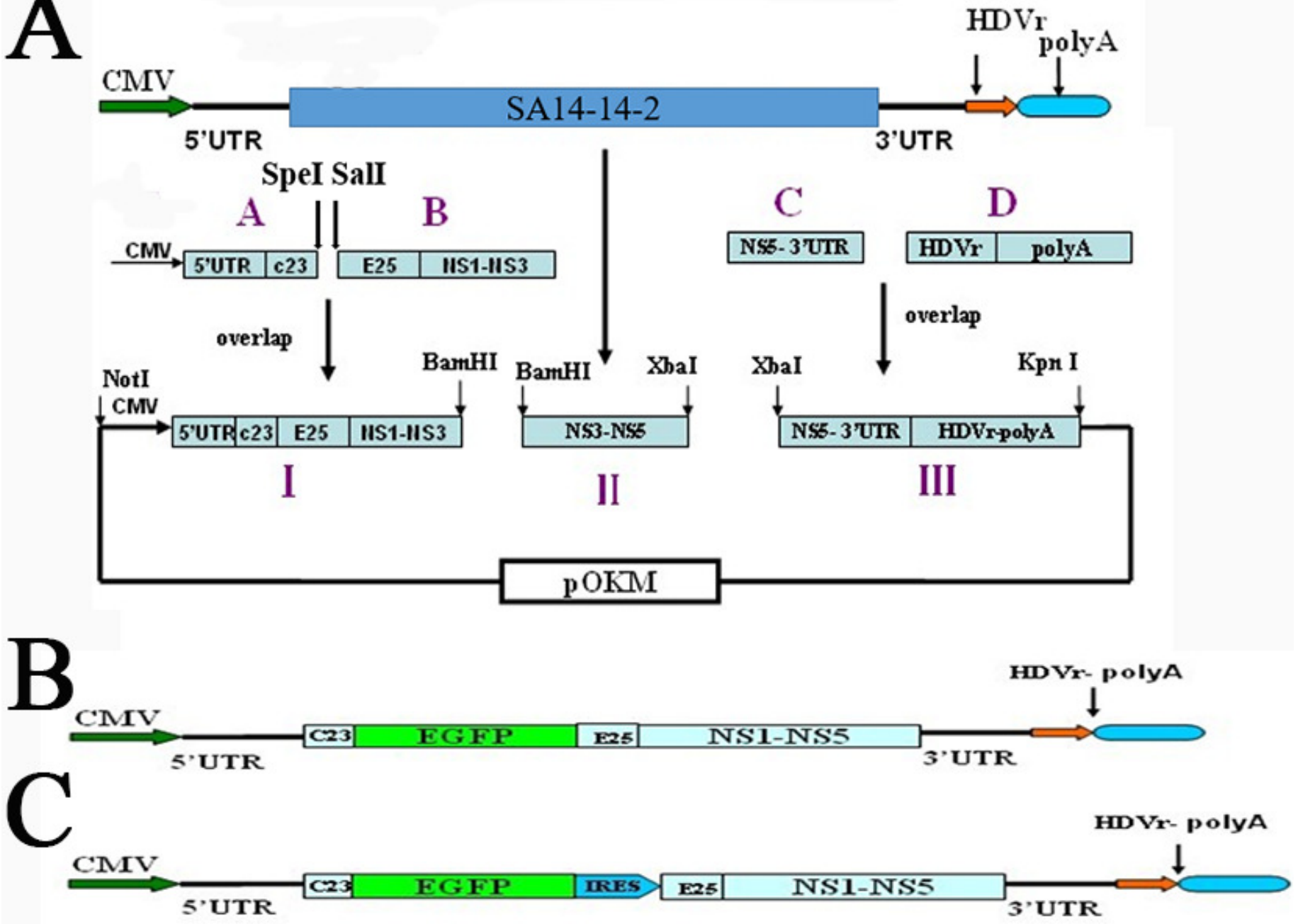


\section{Figure 2}

The efficiency and replication of JEV replicons.

(A) 293 T cells transfected with replicon plasmids pJEV-REP-GFP-IRES or pJEV-REP-GFP were subjected to IFA at the indicated time points post transfection. JEV-NS1 polyclonal antibody and FITC-conjugated goat anti-mouse IgG antibody were used as primary and secondary antibodies for IFA, respectively. (B) BHK-21 cells and 293T cells were transfected with pJEVREP-GFP-IRES or pJEV-REP-GFP and monitored the EGFP signals by fluorescence microscope at $48 \mathrm{~h}$ post transfection.

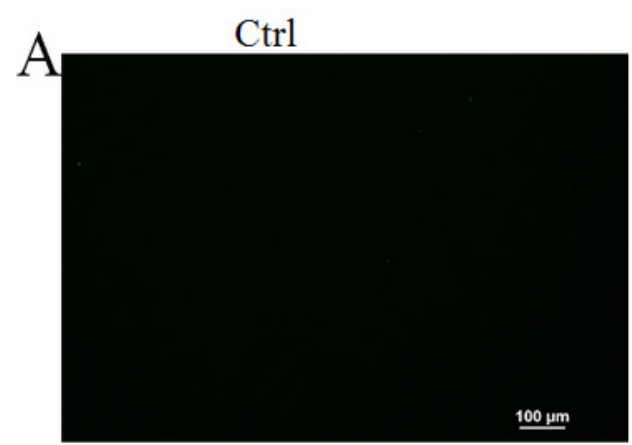

$\mathrm{BHK}-21$

$\mathrm{B}$

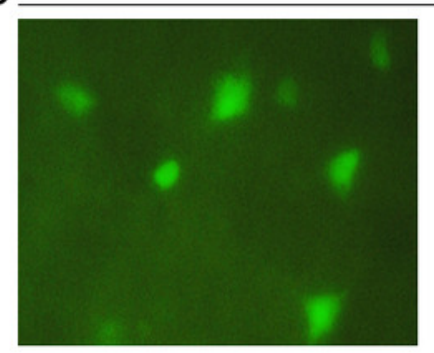

pJEV-REP-GFP

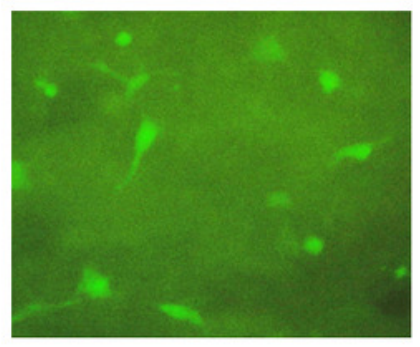

pJEV-REP-GFP-IRES
pJEV-REP-GFP

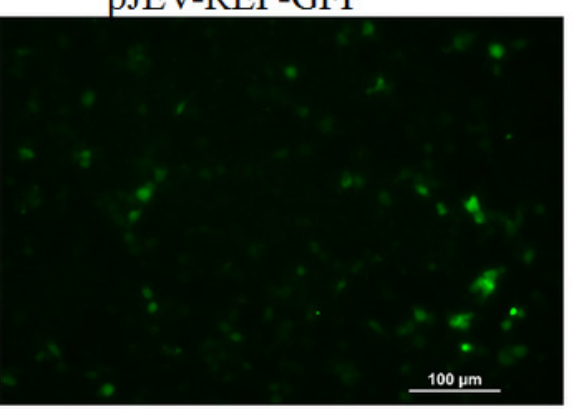

$100 \mu \mathrm{m}$

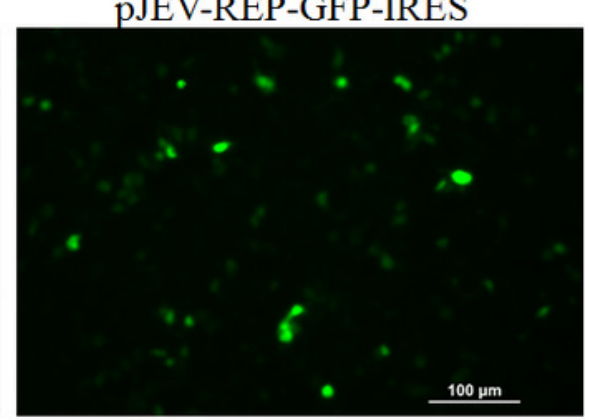

$293 T$

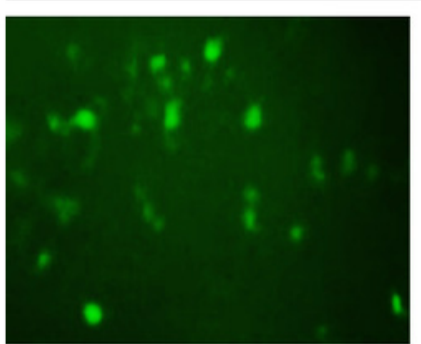

pJEV-REP-GFP

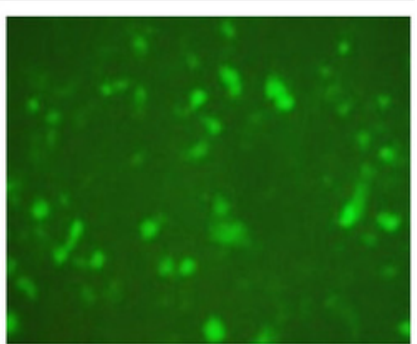

pJEV-REP-GFP-IRES 


\section{Figure 3}

Plus or minus detection of replicon RNA.

(A) BHK-21 cells were transfected with replicon plasmids pJEV-REP-GFP-IRES or pJEV-REP-GFP. Total RNA isolated at $48 \mathrm{~h}$ post transfection was subjected to RT-PCR analysis with JEVspecific primers. RT reaction was performed under standard conditions with avian myeloblastosis virus (AMV) reverse transcriptase (Takara), Primer 2403F and 2609R targeting a region within the $\mathrm{E}$ gene, was used as a sense or an antisense primer respectively in RT step to selectively quantitate either minus- or plus-sense replicon RNA. (B) The cDNA samples obtained from B were diluted with 10 times or 20 times and quantitate either minusor plus-sense replicon RNA by PCR.
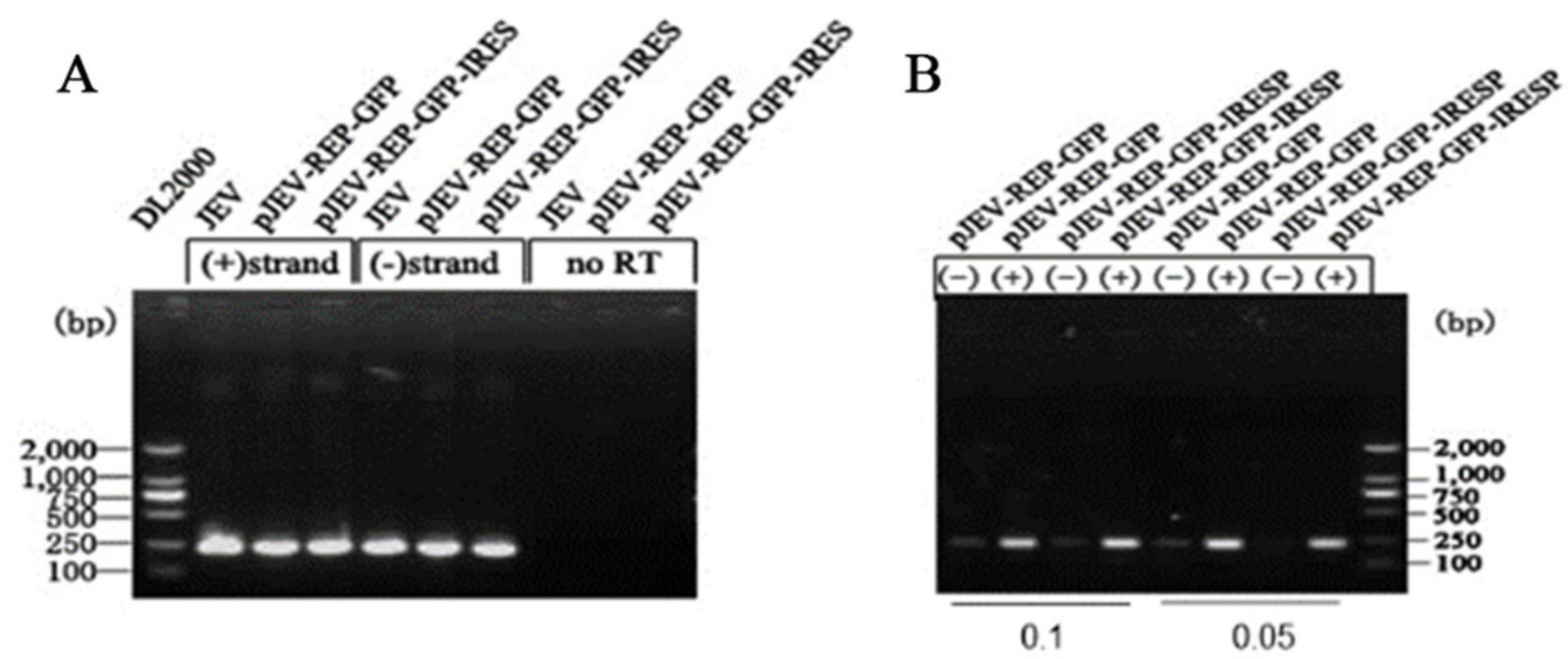


\section{Figure 4}

Expression of PRRSV GP5/M protein in JEV replicons

(A) Schematic drawing of pJEV-REP-G-2A-M and pJEV-REP-G-2A-M-IRES. (B) 293T cells transfected with replicon plasmids pJEV-REP-G-2A-M and pJEV-REP-G-2A-M-IRES were subjected to IFA at the indicated time points post transfection. JEV-NS1 polyclonal antibody and FITC-conjugated goat anti-mouse IgG antibody were used as primary and secondary antibodies for IFA (C) Western Blot was used to analyze the GP5 and M protein expression, PRRSV GP5 protein monoclonal antibodies were used as primary antibodies and IRDye 800conjugated goat anti-mouse as the secondary antibody. 293T cells transfected with pCAGGSGM was used as a positive control while 293 T cells were used as a negative control. in the pJEV-REP-G-2A-M, pJEV-REP-G-2A-M-IRES and pCAGGS-GM, the fusion protein were expressed in $293 T$ cells successfully and in negative control the fusion protein were not detected. 


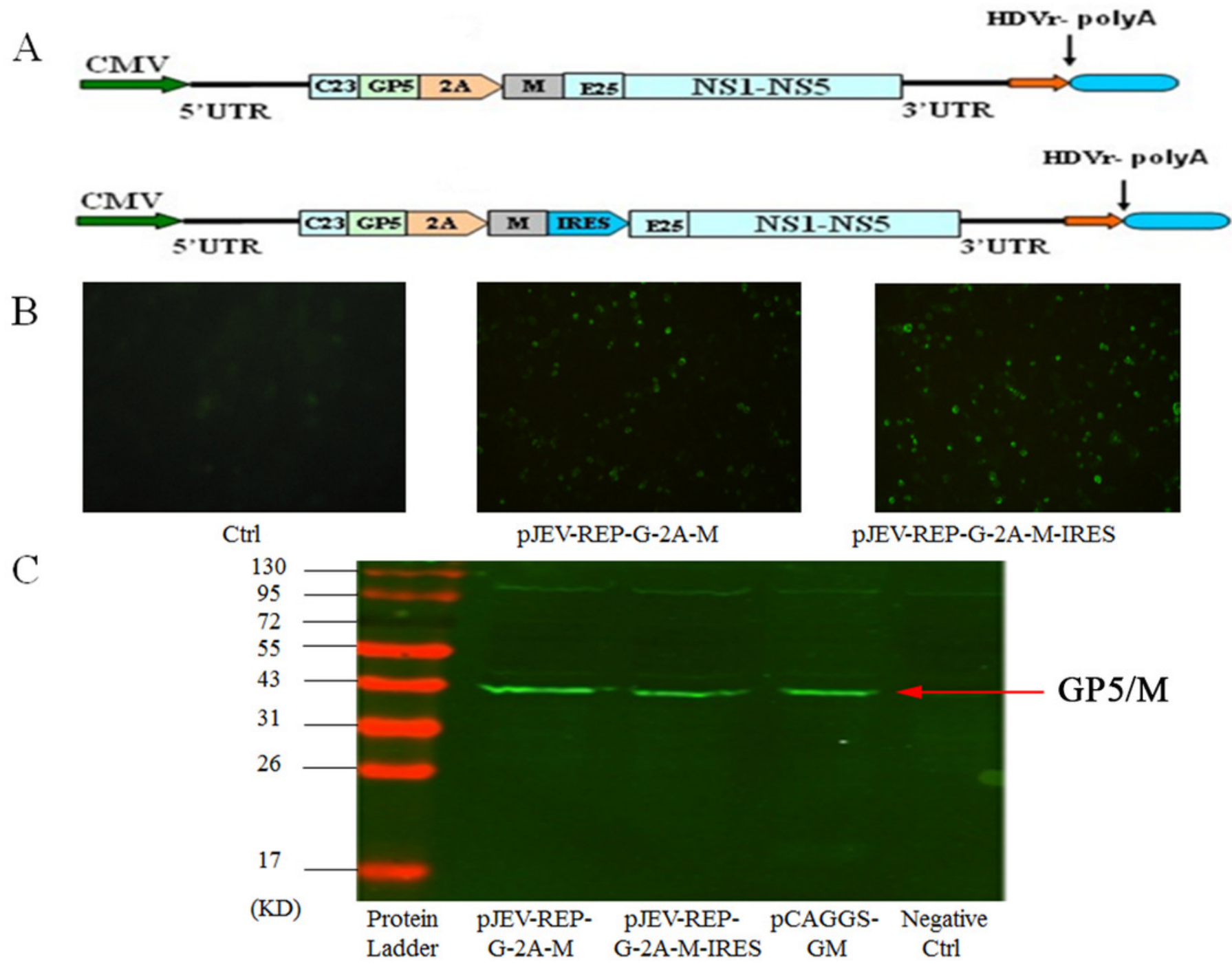




\section{Figure 5}

Lymphocyte proliferation elicited by recombinant JEV replicons expressing GP5/M protein in mice.

Eight-week-old, SPF BALB/c mice were immunized with pJEV-REP-G-2A-M-IRES, pJEV-REP-G2A-M, pCAGGS-GM, pJEV-REP-IRES, and PBS respectively. Lymphocytes were separated from the spleens of mice two weeks after the third immunization. Then, $100 \mu l$ lymphocytes were

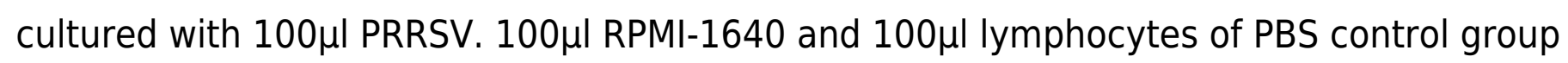
were added into another 96 -well plate as a control. After $84 \mathrm{~h}$, CCK-8 assay was performed to detect the lymphocyte proliferation. Stimulation Index $(\mathrm{SI})=\mathrm{OD}_{450} \mathrm{~nm}$ (PRRSV)/OD ${ }_{450} \mathrm{~nm}$ (Control). Statistical were compared the pCAGGS-GM, pJEV-REP-G-2A-M-IRES and pJEV-REP-G-2A-M groups with the pJEV-REP-IRES group, respectively. 


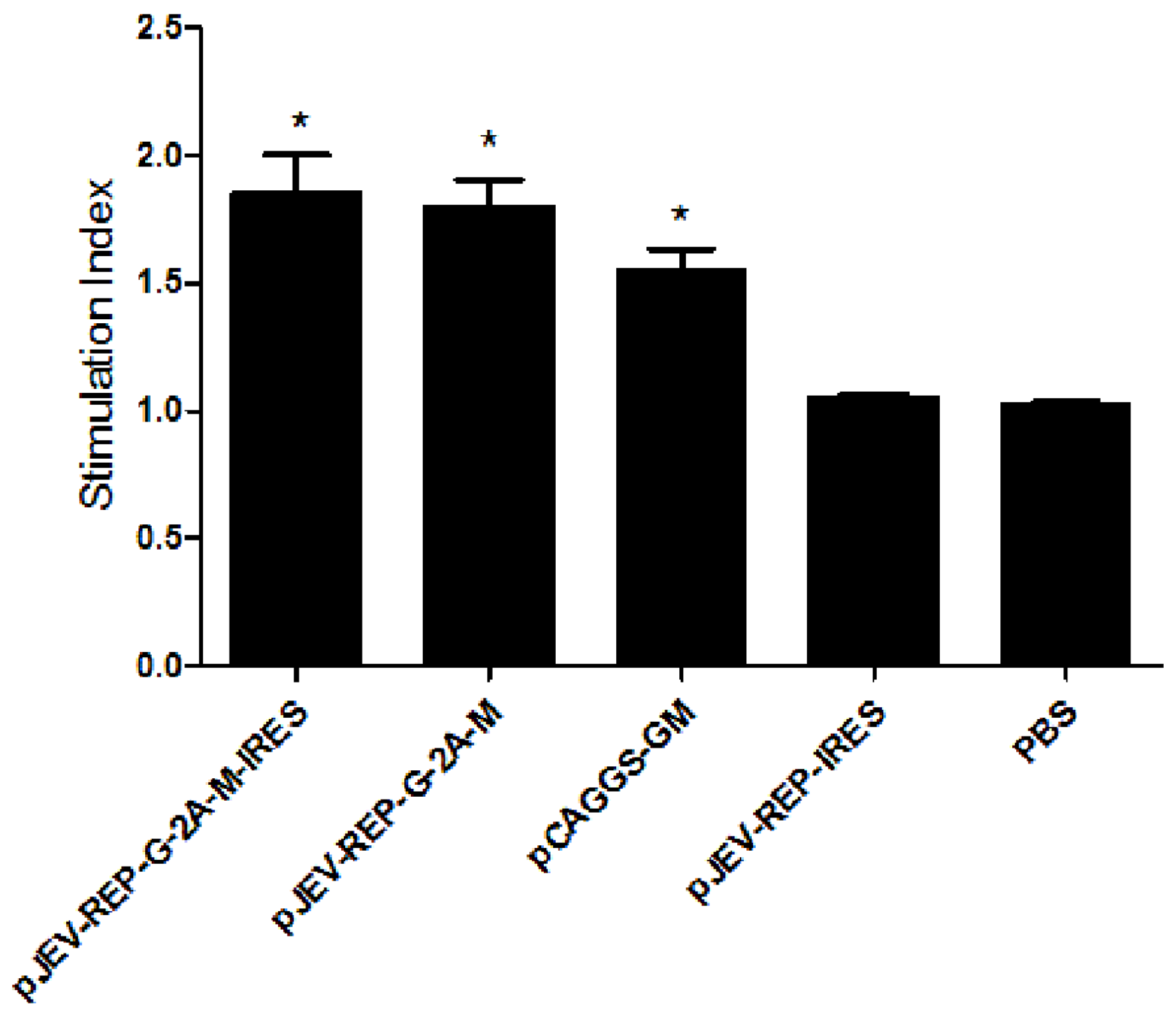


Figure 6

Specific antibodies elicited by recombinant JEV replicons expressing GP5/M protein in mice.

Eight-week-old, SPF BALB/c mice were immunized with pJEV-REP-G-2A-M-IRES, pJEV-REP-G2A-M, pCAGGS-GM, pJEV-REP-IRES, and PBS respectively. Mice sera were collected at indicated time points after immunization and used to detect the GP5 specific antibodies by ELISA.

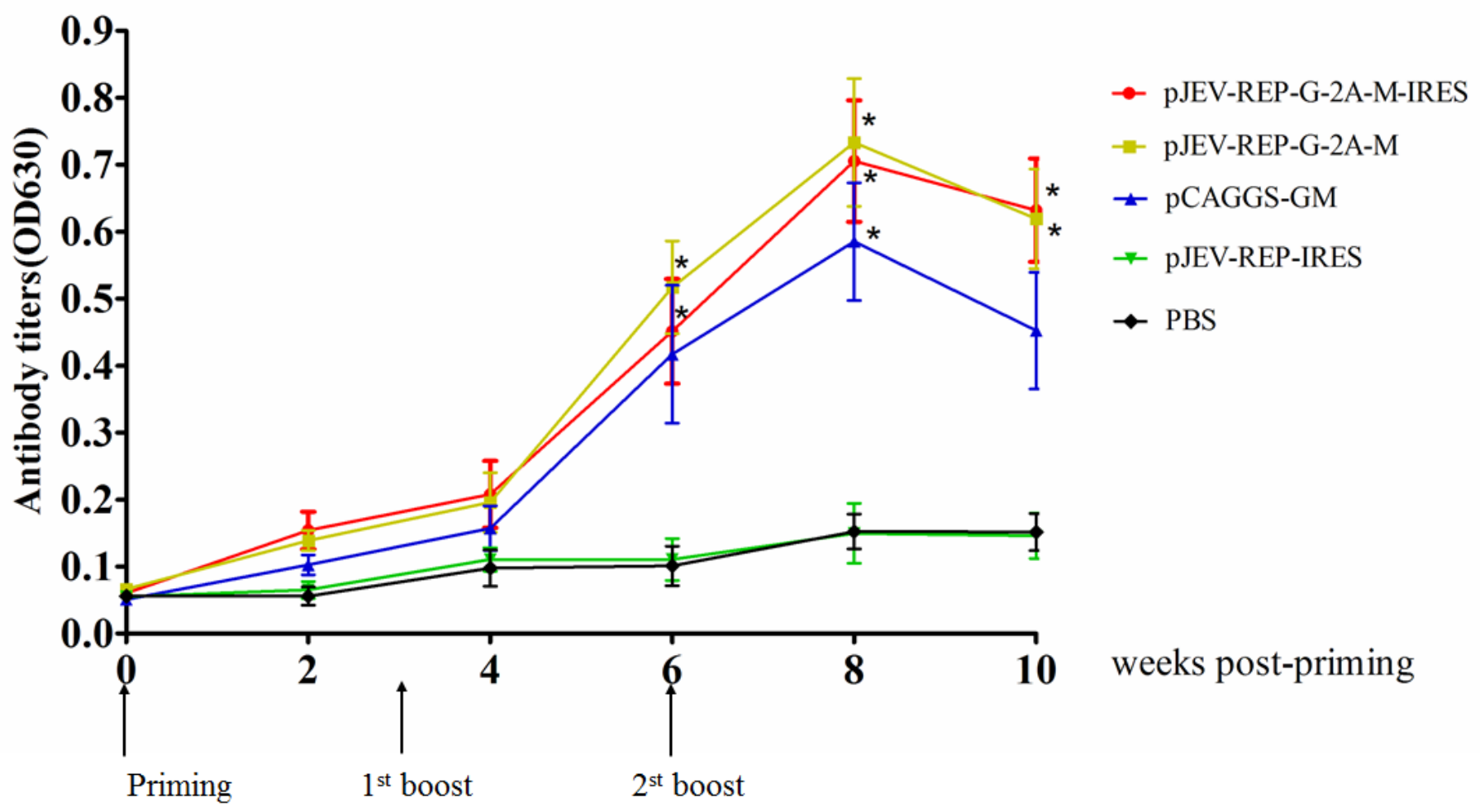

